\title{
Repeatability and reproducibility of optic nerve head perfusion measurements using optical coherence tomography angiography
}

Chieh-Li Chen

Karine D. Bojikian

Chen Xin

Joanne C. Wen

Divakar Gupta

Qinqin Zhang

Raghu C. Mudumbai

Murray A. Johnstone

Philip P. Chen

Ruikang K. Wang 


\title{
Repeatability and reproducibility of optic nerve head perfusion measurements using optical coherence tomography angiography
}

\author{
Chieh-Li Chen, ${ }^{\mathrm{a}, \mathrm{b}}$ Karine D. Bojikian, ${ }^{\mathrm{b}}$ Chen Xin, ${ }^{\mathrm{a}}$ Joanne C. Wen, ${ }^{\mathrm{b}}$ Divakar Gupta, ${ }^{\mathrm{b}}$ Qinqin Zhang, ${ }^{\mathrm{a}}$ \\ Raghu C. Mudumbai, ${ }^{b}$ Murray A. Johnstone, ${ }^{b}$ Philip P. Chen, ${ }^{b}$ and Ruikang K. Wang ${ }^{a, b, *}$ \\ aUniversity of Washington, Department of Bioengineering, 3720 15th Avenue NE, Seattle, Washington 98195, United States \\ bUniversity of Washington, Department of Ophthalmology, 908 Jefferson Street, Seattle, Washington 98104, United States
}

\begin{abstract}
Optical coherence tomography angiography (OCTA) has increasingly become a clinically useful technique in ophthalmic imaging. We evaluate the repeatability and reproducibility of blood perfusion in the optic nerve head $(\mathrm{ONH})$ measured using optical microangiography (OMAG)-based OCTA. Ten eyes from 10 healthy volunteers are recruited and scanned three times with a 68-kHz Cirrus HD-OCT 5000-based OMAG prototype system (Carl Zeiss Meditec Inc., Dublin, California) centered at the ONH involving two separate visits within six weeks. Vascular images are generated with OMAG processing by detecting the differences in OCT signals between consecutive B-scans acquired at the same retina location. ONH perfusion is quantified as flux, vessel area density, and normalized flux within the $\mathrm{ONH}$ for the prelaminar, lamina cribrosa, and the full $\mathrm{ONH}$. Coefficient of variation (CV) and intraclass correlation coefficient (ICC) are used to evaluate intravisit and intervisit repeatability, and interobserver reproducibility. ONH perfusion measurements show high repeatability $[C V \leq 3.7 \%$ (intravisit) and $\leq 5.2 \%$ (intervisit)] and interobserver reproducibility (ICC $\leq 0.966$ ) in all three layers by three metrics. OCTA provides a noninvasive method to visualize and quantify ONH perfusion in human eyes with excellent repeatability and reproducibility, which may add additional insight into ONH perfusion in clinical practice. (C) The Authors. Published by SPIE under a Creative Commons Attribution 3.0 Unported License. Distribution or reproduction of this work in whole or in part requires full attribution of the original publication, including its DOI. [DOI: 10.1117/1.JBO.21.6.065002]
\end{abstract}

Keywords: optical coherence tomography-based angiography; optic nerve head; perfusion; repeatability and reproducibility. Paper 160096R received Feb. 16, 2016; accepted for publication May 19, 2016; published online Jun. 8, 2016.

\section{Introduction}

The disruption of mechanisms that regulate perfusion of ocular tissues has been recognized as an important contributor to the pathogenesis and development of a variety of ocular diseases. ${ }^{1}$ Such diseases include: glaucoma, ${ }^{2-5}$ age-related macular degeneration, ${ }^{6}$ and diabetic retinopathy. ${ }^{7}$ Over the past several decades, various imaging techniques, such as fluorescein angiography, ${ }^{8}$ laser Doppler flowmeter (LDF), ${ }^{2,9,10}$ scanning laser Doppler flowmetry (SLDF), ${ }^{11-16}$ laser speckle flowgraphy (LSFG), ${ }^{17,18}$ and Doppler optical coherence tomography (DOCT), ${ }^{19}$ have been used to directly measure the ocular blood flow and vascular volume, or to indirectly quantify the parameters via perfusion time, flow velocity, flux of the blood cells, or vessel diameter changes.

Although several studies have shown that these techniques are capable of quantifying ocular tissue perfusion, large variations in their measurements limit their application and use in ophthalmology. With SLDF [Heidelberg retina flowmeter (HRF), Heidelberg Engineering, Heidelberg, Germany], the coefficients of variation (CVs) in the literature range from $4 \%$ to $28 \% .^{11,20,21}$ Pemp et al. used an LDF to measure the optic nerve head $(\mathrm{ONH})$ blood flow by detecting a frequency shift of the scattered light according to the optical Doppler effect, ${ }^{9}$ and found that the CVs were $5.9 \%$ for normal subjects and $8.6 \%$ for glaucoma subjects. ${ }^{22}$ A later study from the same

*Address all correspondence to: Ruikang K. Wang, E-mail: wangrk@uw.edu group measured the ocular hemodynamic parameters from 16 healthy subjects using SLDF, the blue field entoptic technique, LDF, laser Doppler velocimetry, retinal vessel analyzer, and color Doppler imaging. The variability from five consecutive measurements was analyzed with each technique; it was found that the CVs of the hemodynamic parameters were between $1.6 \%$ and $18.5 \% .^{23}$ Using LSFG (Kyushu Institute of Technology, Iizuka, Japan), Yaoeda et al. investigated the microcirculation in $\mathrm{ONH}$ from 60 normal volunteers by measuring the square blur rate, volume, flow, and velocity. They reported that the CVs for intervisit repeatability of the parameters ranged from $9.7 \%$ to $19.8 \% .^{17,18}$

We previously developed a noninvasive imaging technique capable of providing three-dimensional in vivo blood flow visualization within microcirculatory tissue beds in the eye. The imaging approach uses a novel optical microangiography (OMAG) technique based on Fourier-domain optical coherence tomography (FD-OCT) systems. ${ }^{24-29}$ By detecting the differences in the scattered light from moving particles, e.g., the red blood cells (RBCs) in vessels, OMAG enables the visualization of microcirculation within tissue beds in the eye. ${ }^{28-30}$ Results with the OMAG technique show promise in the visualization of vessel networks and neovascularization in disease states. ${ }^{24,26,27,29,30}$ Furthermore, we have shown that the OMAG signal is proportional to the concentration of blood flow, which can be referred to as blood flux, i.e., the number of blood cells passing through a vessel cross-sectional area per unit time, ${ }^{31,32}$ and therefore, the blood flux signal permits OMAG to quantify retinal blood. The 
purpose of this study was to evaluate the intravisit and intervisit repeatability as well as the interobserver reproducibility of $\mathrm{ONH}$ perfusion measurements using the OMAG technique.

\section{Methods}

\subsection{Subjects}

This study was approved by the Institutional Review Board of the University of Washington (UW) and informed consent was obtained from all subjects. This study followed the tenets of the Declaration of Helsinki and was conducted in compliance with the Health Insurance Portability and Accountability Act.

Volunteers with normal optic discs were prospectively recruited at the UW Eye Institute. Inclusion criteria were best-corrected visual acuity of $20 / 40$ or better, refractive error between -6 and $+3 \mathrm{D}$ spherical equivalent, and a normal retinal nerve fiber layer (RNFL) thickness on FD-OCT. Exclusion criteria were significant media opacity preventing high-quality imaging, previous diagnosis of migraine, or any ocular disease. The right eye from each subject was imaged and included in this study.

\subsection{Image Acquisition and Scanning Protocol}

All eyes were scanned using a 68-kHz Cirrus HD-OCT 5000based OMAG prototype spectral-domain (SD-) OCT system with active motion-tracking capability (Carl Zeiss Meditec Inc., Dublin, California) to minimize motion artifacts. ${ }^{33}$ The center wavelength of the OCT system worked at $840 \mathrm{~nm}$ with a typical $3-\mathrm{dB}$ bandwidth of $45 \mathrm{~nm}$, axial resolution of $8 \mu \mathrm{m}$, and lateral resolution of $15 \mu \mathrm{m}$. Three OMAG scans centered on the $\mathrm{ONH}$ were acquired at each visit. Between each scan, patients were asked to sit back and rest for about $30 \mathrm{~s}$ to 1 min. Participants returned for a second visit within a 6week time frame, at which time the same scanning protocol was repeated by the same operator.

The OMAG scan pattern generated a volumetric dataset over a $2.4 \times 2.4 \mathrm{~mm}^{2}$ area centered on the optic disc. Each single Bscan was composed of 245 A-scans. For each A-scan, 1024 sampling points were collected along a $2.0-\mathrm{mm}$ axial scan depth. Four consecutive B-scans were acquired at each fixed transverse location (i.e., along the fast axis) before the scanning probe moved to the next transverse location on the optic disc. A total of 245 transverse locations were sampled, located $\sim 9.8 \mu \mathrm{m}$ apart. The time difference between two successive B-scans was $\sim 3.6 \mathrm{~ms}$, which corresponds to a B-scan acquisition rate of $270 \mathrm{~B}$-scans/s.

\subsection{OMAG Processing}

All acquired volumetric scans were processed with an OMAG algorithm to extract both the structural and blood flow signals. The details of the OMAG algorithm have been described elsewhere. ${ }^{28,29}$ Briefly, blood flow signals were calculated by subtracting complex OCT signals between each consecutive B-scan pair at each location. ${ }^{28,29}$ The intensity of the blood flow signal is proportional to the amount of RBCs passing through the vessels. ${ }^{31,32}$ The average of the differences at each location was calculated to generate the final blood flow signal, $I_{\text {flow }}(x, z)$, as described in

$I_{\text {flow }}(x, z)=\frac{1}{R-1} \sum_{i=1}^{R-1}\left|C_{i+1}(x, z)-C_{i}(x, z)\right|$, where $i$ is the index of the repeated time of B-scans at each transverse location, $C(x, z)$ indicates the complex OCT signal (including both phase and amplitude information) at the $x^{\prime}$ th A-scan of the B-scan and $z$ 'th sampling point in the axial direction. The number of repeated $\mathrm{B}$-scan, $R$, equals 4 in the current imaging protocol for higher signal-to-noise ratio of the flow signal.

\subsection{Retinal Layer Segmentation and Vascular En Face Images}

Using the structural images, boundaries of the inner limiting membrane (ILM), the anterior surface of lamina cribrosa (aLC), and the visible posterior surface of lamina cribrosa (pLC) were delineated using a laboratory-developed semiautomatic retinal layer segmentation program. ${ }^{34}$ The segmentation software automatically detected the boundaries of the retinal layers from the structural OCT cross-sectional images by measuring the gradient of OCT signals. ${ }^{34}$ When the changes of intensity were too indistinct for the algorithm to detect a boundary, the operator could interrupt the automatic algorithm and manually guide the system to identify the correct boundaries. Three retinal layers were defined based on the segmented retinal boundaries: the prelaminar tissue [preLC, between ILM and aLC, Fig. 1(a)], lamina cribrosa (LC, between aLC and pLC), and full $\mathrm{ONH}$ (between ILM and pLC). Since vascular images were derived from the structural images, the boundaries detected based on the structural image could then be applied to OMAG vascular images [Fig. 1(b)] to provide precise correspondence between structural and OMAG retinal layer locations.

Retinal layer segmentation was performed on all the images by a single operator [Chieh-Li Chen (CLC)]. To further test the interobserver reproducibility, two independent operators (CLC and $\mathrm{CX}$ ) performed the layer segmentation in a masked manner with the same program on one randomly selected scan for each eye.

Maximum intensity projection analyses, which detect the blood flow signal with the highest intensity value along each A-scan, were performed within each segmented retinal layer to generate vascular en face images of preLC, LC, and the full $\mathrm{ONH}$. The boundary of the optic disc margin to be used for later analysis was manually delineated by identifying the edge of Bruch's membrane on the structural B-scans.

\subsection{Flux, Vessel Area Density, and Normalized Flux Measurements}

To quantify $\mathrm{ONH}$ perfusion, we created three quantitative measurements: flux, vessel area density, and normalized flux. ${ }^{35}$

The OMAG technique measured the variations in OCT signals between consecutive B-scans by calculating the differences in complex OCT signals. The differences in the complex OCT signals contained both differences from phase and amplitude information. The phase differences resulted from the motion of RBCs flowing through the vessels, whereas the amplitude differences came from the variation of the scattered OCT signal and was related to the number of particles passing through the vessels. Combining both information of flow velocity and the number of particles (concentration), the intensity of flow signal is an indicator of flux (the number of particles passing through the cross section per unit time), and we first measured the flux using 

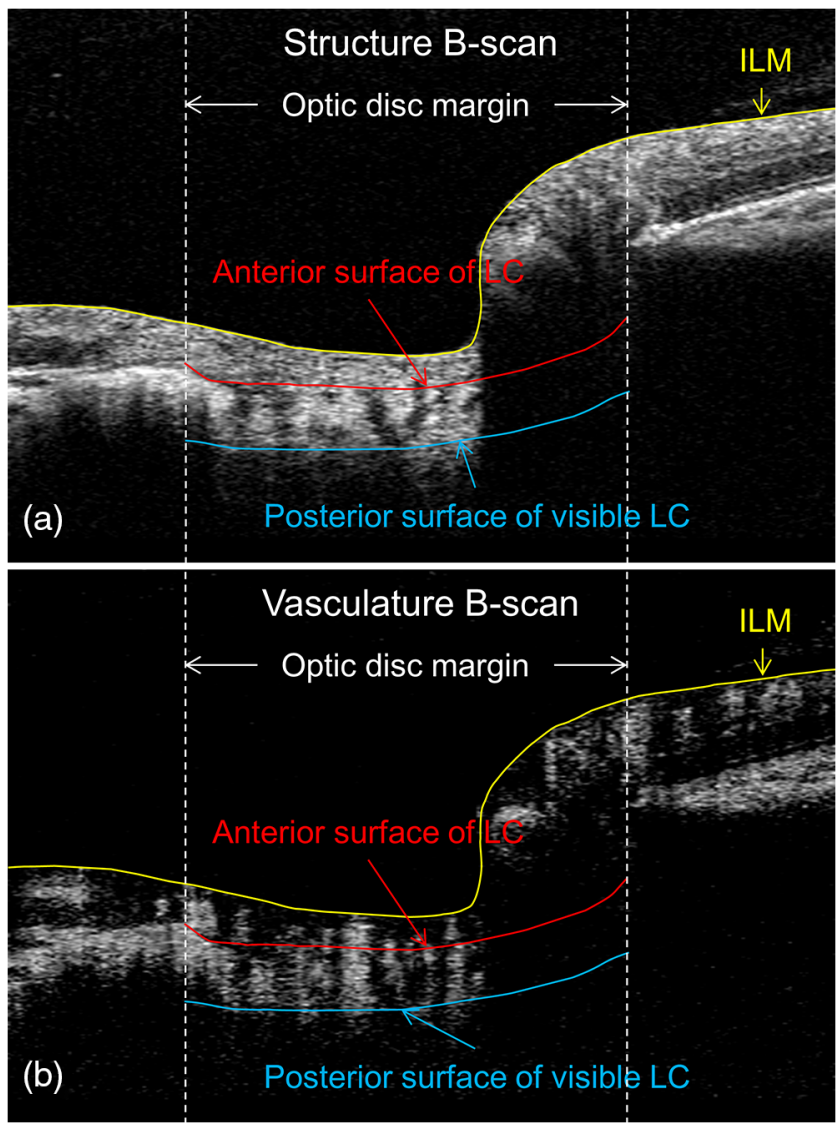

Fig. 1 Example of retinal layer segmentation. Three retinal boundaries were segmented: ILM (the yellow line), anterior surface of LC (the red line within the optic disc), and posterior surface of visible LC (the blue line). (a) and (b) Structural and vascular cross-sectional images superimposed with segmented retinal boundaries.

flux $=\frac{\sum_{(x, y) \in \mathrm{ONH}} \frac{I_{\text {flow }}(x, y)}{255}}{\operatorname{area}_{\mathrm{ONH}}}, \quad$ where area $\mathrm{ONH}=\sum_{(x, y) \in \mathrm{ONH}} 1$,

where the blood flow signal $I_{\text {flow }}$ was normalized to between 0 and 1 by dividing the measured value by 255 (the full dynamic range of the flow signal); the calculation resulted in a unit-less ratio useful for relative blood flow comparisons between discs.

Vessel area density was the ratio of vessel-containing area within the ONH $v(x, y)=1$ to the total $\mathrm{ONH}$ area [Eq. (3)]. Vascular en face images of the blood vessels in the $\mathrm{ONH}$ were detected by a multiscale Hessian filter developed based on the method of Frangi et al. ${ }^{36}$ The Hessian filter measures the local curvature by calculating the second-order partial derivatives of local structure from the vascular en face images. Based on the calculated two-dimensional eigenvalues $\lambda_{1}$ and $\lambda_{2}$, where $\left|\lambda_{1}\right| \leq\left|\lambda_{2}\right|$, the structural information was converted into probability-like estimates of vessel presence ${ }^{36}$

$$
\begin{gathered}
\text { vessel area density }=\frac{\sum_{(x, y) \in \mathrm{ONH}} v(x, y)}{\text { area }_{\mathrm{ONH}}}, \\
v(x, y)=\left\{\begin{array}{ll}
1, & \text { if }(x, y) \text { is a vessel } \\
0, & \text { if }(x, y) \text { is not a vessel }
\end{array} .\right.
\end{gathered}
$$

As less nerve head tissue requires less ONH perfusion, we further calculated the normalized flux to avoid bias from the reduced vessel area, e.g., in the situation of comparing $\mathrm{ONH}$ perfusion between glaucomatous and normal eyes. In Eq. (4), the flow signals accumulated from the vessels were divided by the vessel area, indicating the evaluation of flux in the vessels only:

$$
\begin{gathered}
\text { normalized flux }=\frac{\sum_{(x, y) \in \mathrm{ONH}} \frac{I_{\mathrm{flow}}(x, y) \times v(x, y)}{255}}{\sum_{(x, y) \in \mathrm{ONH}} v(x, y)}, \\
v(x, y)= \begin{cases}1, & \text { if }(x, y) \text { is a vessel } \\
0, & \text { if }(x, y) \text { is not a vessel }\end{cases}
\end{gathered}
$$

Similar to the flux, in Eq. (4), the blood flow signal was normalized to between 0 and 1 by dividing measured flow by the full dynamic range of blood flow signal intensity yielding a unit-less number that again permits comparison between discs.

\subsection{Statistical Analysis}

Demographic and clinical characteristics were summarized by standard descriptive statistics. For each eye, two CVs were calculated from three scans (acquired at the same visit) and six scans (from two visits) to evaluate the intravisit and intervisit repeatability, respectively, for all three $\mathrm{ONH}$ perfusion metrics. Mean CVs and standard deviations of 10 subjects were further computed to represent the final intravisit and intervisit repeatability for each $\mathrm{ONH}$ perfusion metric. To evaluate agreement between the observers' measurements for interobserver reproducibility, the intraclass correlation coefficient (ICC) two-way mixed model with their $95 \%$ confidence interval on absolute agreement was used to analyze measurement reliability. ${ }^{37}$ The values of the ICC can range from 0 to 1 , with a higher value indicating better reliability. ICC less than 0.40 was considered as poor, values between 0.4 and 0.6 as fair, values between 0.61 and 0.8 as good, and values higher than 0.8 as excellent. ${ }^{38}$ IBM SPSS Statistics for Windows, Version 23.0 (IBM Corp., Armonk, New York) was used for statistical analyses.

\section{Results}

Ten eyes from 10 healthy volunteers (five men and five women) were recruited at the UW Eye Institute. The mean age was $35.9 \pm 8.6$-years old (range 28.8 to 50.0-years old). The mean RNFL thickness of the normal subjects was $97.7 \pm 6.6 \mu \mathrm{m}$. All the scans were included in the analysis.

Figure 2 shows an example of the vascular en face images and vessel maps detected using a Hessian filter of preLC, LC, and full ONH from a 28 -year-old healthy woman. Three scans acquired at the same visit are displayed in adjacent images. Excellent intravisit repeatability of vascular networks in the ONH can be observed. In addition to the central retinal arteries and veins, the capillaries inside and surrounding the $\mathrm{ONH}$ were also visualized.

Analysis of the measurements of three $\mathrm{ONH}$ perfusion metrics evaluating intravisit repeatability and the corresponding CVs for the three retinal layers are listed in Table 1. In all retinal layers, vessel area density showed the best intravisit repeatability, followed by normalized flux, and finally by flux.

Table 2 summarizes the intervisit repeatability for the three $\mathrm{ONH}$ perfusion metrics. In preLC and full ONH, vessel area density demonstrated the highest intervisit repeatability, 

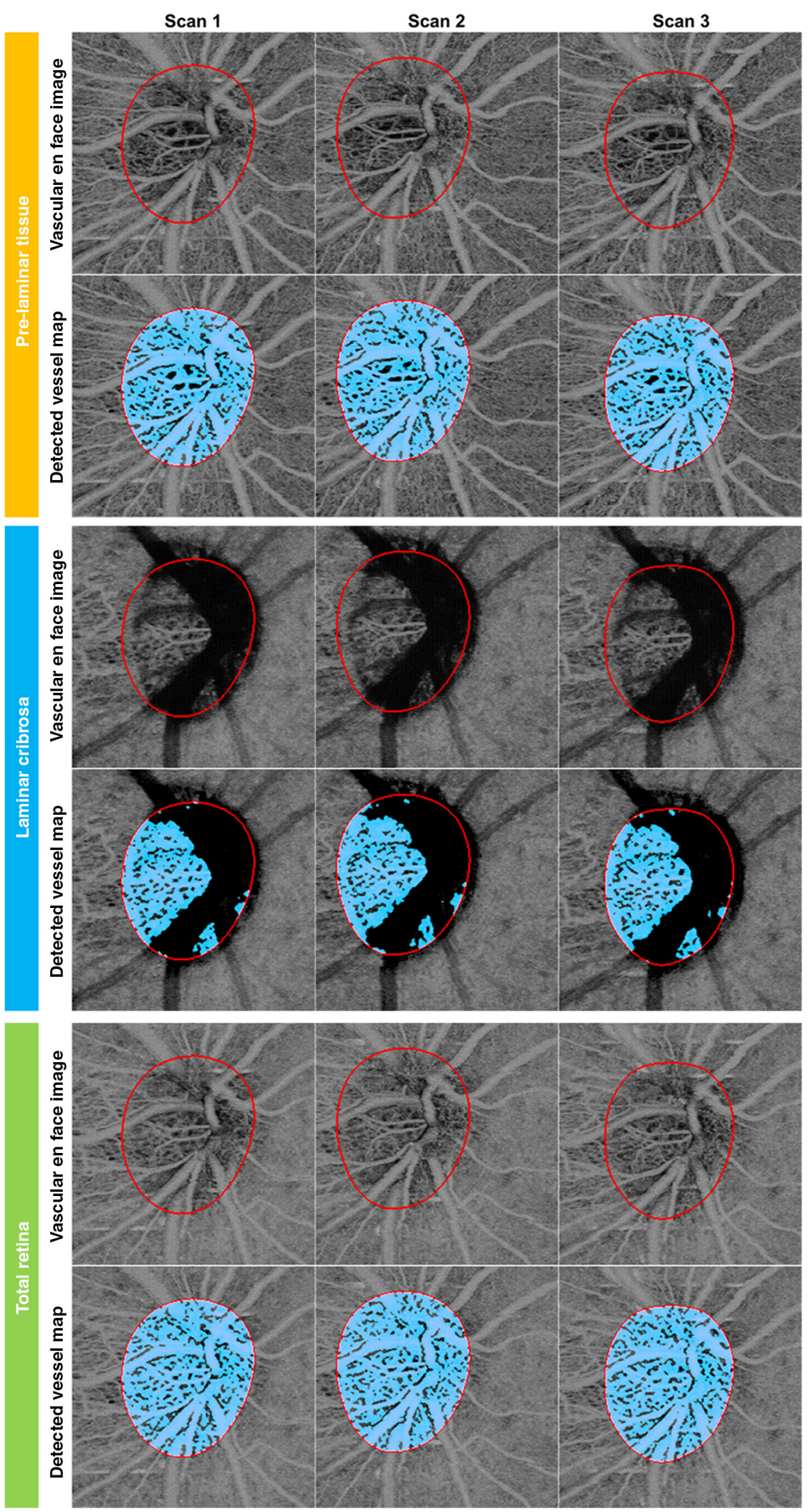

Fig. 2 An example result of the vascular en face images and detected vessel maps of prelaminar layer (preLC, top, orange margin), lamina cribrosa (LC, middle, blue margin), and full ONH (bottom, green margin) of a normal eye. Three scans acquired on the same visit were displayed adjacently. For each layer, the vascular en face images are presented in the top row and detected vessel map is shown in the bottom row. 
Table 1 Intravisit repeatability of ONH perfusion metrics (flux, vessel area density, and normalized flux) using OCTA in normal eyes.

\begin{tabular}{lccccrc} 
& \multicolumn{4}{c}{ Intravisit repeatability } \\
\cline { 2 - 6 } & Flux & CV (\%) & Vessel area density & CV (\%) & Normalized flux & CV (\%) \\
\hline Prelaminar tissue & $0.35 \pm 0.04$ & $3.7 \pm 2.4$ & $0.78 \pm 0.02$ & $0.6 \pm 0.3$ & $0.38 \pm 0.04$ & $3.3 \pm 2.2$ \\
Lamina cribrosa & $0.14 \pm 0.05$ & $3.7 \pm 2.3$ & $0.39 \pm 0.13$ & $3.0 \pm 3.0$ & $0.26 \pm 0.04$ & $3.4 \pm 3.0$ \\
Full ONH & $0.36 \pm 0.04$ & $3.3 \pm 2.3$ & $0.78 \pm 0.01$ & $0.8 \pm 0.3$ & $0.39 \pm 0.04$ & $3.0 \pm 2.1$ \\
\hline
\end{tabular}

Note: $\mathrm{CV}$, coefficient of variation. $\mathrm{ONH}$ perfusion metrics and $\mathrm{CVs}$ are presented as mean \pm standard deviation.

Table 2 Intervisit repeatability of ONH perfusion metrics (flux, vessel area density, and normalized flux) using OCTA in normal eyes.

\begin{tabular}{lcccccc}
\hline & \multicolumn{5}{c}{ Intervisit repeatability } \\
\cline { 2 - 7 } & Flux & CV (\%) & Vessel area density & CV (\%) & Normalized flux & CV (\%) \\
\hline Prelaminar tissue & $0.35 \pm 0.04$ & $4.8 \pm 1.5$ & $0.78 \pm 0.02$ & $0.9 \pm 0.3$ & $0.38 \pm 0.04$ & $4.2 \pm 1.3$ \\
Lamina cribrosa & $0.14 \pm 0.05$ & $4.8 \pm 2.1$ & $0.38 \pm 0.13$ & $5.2 \pm 3.3$ & $0.26 \pm 0.04$ & $3.7 \pm 1.7$ \\
Full ONH & $0.36 \pm 0.03$ & $4.4 \pm 1.5$ & $0.78 \pm 0.01$ & $1.0 \pm 0.3$ & $0.39 \pm 0.03$ & $3.7 \pm 1.3$ \\
\hline
\end{tabular}

Note: $\mathrm{CV}$, coefficient of variation. ONH perfusion metrics and CVs are presented as mean \pm standard deviation.

followed by normalized flux, and then by flux, whereas in the LC, normalized flux showed the highest intervisit repeatability, followed by flux; vessel area density had the lowest intervisit repeatability.

Interobserver reproducibility of $\mathrm{ONH}$ perfusion measurements was analyzed as ICC and its 95th percentile confidence intervals, which are presented in Table 3 . For the interobserver reproducibility, all three $\mathrm{ONH}$ perfusion metrics showed excellent agreement between observers in each of the three retinal layers: all measurements were above $0.950 .{ }^{38}$

\section{Discussion}

We demonstrate that OMAG enables visualization of the microcirculation in the $\mathrm{ONH}$ and allows the quantification of $\mathrm{ONH}$ perfusion in normal eyes with high repeatability and reproducibility. By detecting the differences in the backscattered OCT signals caused by RBCs between adjacent cross-sectional frames at the same location, OMAG is able to generate blood flow signals with high resolution in a noninvasive fashion. Since the blood flow signals are proportional to the number of RBCs passing through the vessels, ${ }^{31,32}$ the quantification of $\mathrm{ONH}$ perfusion becomes possible.

Various imaging techniques have been developed to measure and quantify the ONH perfusion. However, both repeatability and reproducibility have been considered problematic with all techniques used to date thus limiting their value in clinical practice as well as in clinical trials. ${ }^{11,17,18,20-23}$ In our study, using the OCTA with OMAG technique for quantifying ONH perfusion, the intravisit and intervisit repeatability were $\leq 3.3 \%$ and $\leq 4.4 \%$ (CVs for the full $\mathrm{ONH}$ ). Our results provide $\mathrm{ONH}$ perfusion measurements with higher repeatability and reproducibility values than those from other reports of noninvasive imaging

Table 3 Interobserver reproducibility of ONH perfusion metrics (flux, vessel area density, and normalized flux) using OMAG in normal eyes.

\begin{tabular}{lcccccc} 
& \multicolumn{5}{c}{ Interobserver reproducibility } \\
\cline { 2 - 7 } & Flux & ICC & Vessel area density & ICC & Normalized flux & ICC \\
\hline Prelaminar tissue & $0.35 \pm 0.04$ & 0.994 & $0.78 \pm 0.02$ & 0.987 & $0.38 \pm 0.04$ & 0.994 \\
& & $(0.975,0.998)$ & & $(0.951,0.997)$ & $0.978,0.999)$ \\
Lamina cribrosa & $0.14 \pm 0.05$ & 0.991 & $0.39 \pm 0.14$ & 0.993 & $0.26 \pm 0.04$ & 0.966 \\
& & $(0.965,0.998)$ & & $(0.973,0.998)$ & $0.871,0.991)$ \\
Full ONH & $0.36 \pm 0.04$ & 0.998 & $0.78 \pm 0.01$ & 0.993 & $0.39 \pm 0.04$ & 0.999 \\
& & $(0.998,1.000)$ & & $(0.972,0.998)$ & $(0.971,1.000)$ \\
\hline
\end{tabular}

Note: ICC, intraclass correlation coefficient. ONH perfusion metrics are presented as mean \pm standard deviation. For ICC, $95 \%$ confidence interval is shown in the parentheses. 
techniques. Unlike LSFG, which detects the accumulated speckle changes and measures the blurriness of the images, or LDF, which detects the flow signal by measuring the Doppler frequency shift, OMAG-based OCTA directly measures the localized differences in consecutive OCT signals. In this way, OMAG-based OCTA is more sensitive to the microcirculation in the tissue, and, therefore, is able to generate blood flow images with higher resolution, better contrast, and, as a consequence, less variability.

Two studies have assessed the intravisit and intervisit repeatability of $\mathrm{ONH}$ perfusion using a split-spectrum amplitudedecorrelation angiography (SSADA) algorithm, another OCTA technique. ${ }^{39,40}$ High intravisit $(\leq 1.2 \%$ mean CV) and intervisit repeatability $(\leq 4.2 \%$ mean $\mathrm{CV})$ of $\mathrm{ONH}$ perfusion measurements were detected using OCTA with the SSADA algorithm, further supporting and strengthening the proposal that OCTA-based methods provide reproducible and reliable measurement of $\mathrm{ONH}$ perfusion and may be suitable for clinical practice.

A number of factors could be responsible for the slightly higher $\mathrm{CV}$ values reported in our study compared to others who used SSADA-based OCTA. ${ }^{39,40}$ In the SSADA algorithm, an extra image registration algorithm was required to register 4 orthogonal raster scanned volumes and to remove the motion between B-scan positions along the slow scan axis. ${ }^{39}$ The angle and location of the acquisition can vary between both same day and different day scans. It is conceivable that the additional image registration algorithm used to register the images between scans may unintentionally introduce an apparent reduction in between-scan and between-visit variability. Our OMAG processing compensates for the variation between consecutive B-scans at the same location but not for the variation in the sampling location in the longitudinal direction. Nonetheless, our results demonstrate excellent intravisit and intervisit repeatability and interobserver reproducibility.

Another possible reason for the slightly higher $\mathrm{CV}$ values seen with OMAG-based OCTA is that three subjects were unable to be scanned at the same time of day at two consecutive visits. Since $\mathrm{ONH}$ perfusion fluctuates during the day, ${ }^{21}$ the time difference may contribute to a larger variation in our results. Additionally, differences in the methods of blood flow signal extraction and image segmentation could contribute to variability among studies.

With the use of a light source with a central wavelength at $840 \mathrm{~nm}$ in our prototype SD-OCT system, overlying prelaminar tissue may reduce the visibility of microcirculation in the LC. The intact prelaminar tissue in the $\mathrm{ONH}$ may block the light signal and thus limit our ability to visualize deep tissues. Such a blockage of the light signal may cause increased variation in ONH perfusion measurements in the LC, especially for vessel area density assessment. The limited penetration issue might be resolved by using a light source with a longer center wavelength, or using swept-source OCT at 1060-nm wavelength. ${ }^{41,42}$

One limitation of this study is that we involved only healthy subjects to test the repeatability and reproducibility of ONH perfusion measurements using OCTA with the OMAG technique. Since this was the first study testing the repeatability and reproducibility to quantify ONH perfusion using OMAG, we started with normal subjects to make sure that the flow measurements were not affected by disease progression. Other challenges of having diseased eyes in the repeatability and reproducibility study are that the repeatability and reproducibility may be affected by the larger variations in the flow signal in the defect areas, or receiving treatment. Nevertheless, further investigation with diseased eyes, such as glaucoma or diabetic retinopathy, to reflect the actual clinical situation and to provide better estimation is required.

In conclusion, OMAG provides a noninvasive method to visualize and quantify $\mathrm{ONH}$ perfusion in human eyes with excellent repeatability and reproducibility. Our results show that OMAG may add additional insights into ONH perfusion. Such insights also have the potential to provide a better understanding of the mechanisms and etiology of perfusion abnormalities in eye diseases, monitoring of their progression and also improve decisions in managing their care.

\section{Acknowledgments}

This study was supported in part by the National Institutes of Health contract NEI R01-EY024158, Carl Zeiss Meditec Inc., and Research to Prevent Blindness (New York, New York). C.L.C., Q.Z., and R.K.W. received research support from Carl Zeiss Meditec, Inc. M.A.J. is a consultant for Healonics, Sensimed, Ivantis, Cascade Ophthalmics, and receives royalties from intellectual property owned by Allergan. R.K.W. has significant financial interest in the intellectual property of OMAG technology, owned by Oregon Health \& Science University. K.D.B., C.X., J.C.W., D.G., R.C.M., and P.P.C.: None.

\section{References}

1. G. A. Cioffi and A. Alm, "Measurement of ocular blood flow," J. Glaucoma 10(5 Suppl. 1), S62-S64 (2001).

2. J. E. Grunwald et al., "Optic nerve and choroidal circulation in glaucoma," Invest Ophthalmol. Vis. Sci. 39(12), 2329-2336 (1998).

3. H. S. Chung et al., "Vascular aspects in the pathophysiology of glaucomatous optic neuropathy," Surv. Ophthalmol. 43(Suppl. 1), S43-S50 (1999).

4. H. S. Chung et al., "Peripapillary retinal blood flow in normal tension glaucoma," Br. J. Ophthalmol. 83(4), 466-469 (1999).

5. J. Flammer et al., "The impact of ocular blood flow in glaucoma," Prog. Retinal Eye Res. 21(4), 359-393 (2002).

6. M. R. Hee et al., "Optical coherence tomography of age-related macular degeneration and choroidal neovascularization," Ophthalmology 103(8), 1260-1270 (1996).

7. H. P. Hammes et al., "Diabetic retinopathy: targeting vasoregression," Diabetes 60(1), 9-16 (2011).

8. O. Arend et al., "Capillary density and retinal diameter measurements and their impact on altered retinal circulation in glaucoma: a digital fluorescein angiographic study," Br. J. Ophthalmol. 86(4), 429-433 (2002).

9. C. E. Riva et al., "Laser Doppler flowmetry in the optic nerve," Exp. Eye Res. 55(3), 499-506 (1992).

10. B. L. Petrig, C. E. Riva, and S. S. Hayreh, "Laser Doppler flowmetry and optic nerve head blood flow," Am. J. Ophthalmol. 127(4), 413-425 (1999).

11. G. Hollo, T. J. van den Berg, and E. L. Greve, "Scanning laser Doppler flowmetry in glaucoma," Int. Ophthalmol. 20(1-3), 63-70 (1996).

12. G. Michelson, M. J. Langhans, and M. J. Groh, "Perfusion of the juxtapapillary retina and the neuroretinal rim area in primary open angle glaucoma," J. Glaucoma 5(2), 91-98 (1996).

13. J. Kerr, P. Nelson, and C. O'Brien, "A comparison of ocular blood flow in untreated primary open-angle glaucoma and ocular hypertension," Am. J. Ophthalmol 126(1), 42-51 (1998).

14. G. Fuchsjager-Mayrl et al., "Ocular blood flow and systemic blood pressure in patients with primary open-angle glaucoma and ocular hypertension," Invest. Ophthalmol. Vis. Sci. 45(3), 834-839 (2004).

15. A. Harris et al., "Blood flow per unit retinal nerve fibre tissue volume is lower in the human inferior retina," Br. J. Ophthalmol. 87(2), 184-188 (2003). 
16. E. A. Sato et al., "Decreased blood flow at neuroretinal rim of optic nerve head corresponds with visual field deficit in eyes with normal tension glaucoma," Graefes Arch. Clin. Exp. Ophthalmol. 244(7), 795-801 (2006)

17. K. Yaoeda et al., "Measurement of microcirculation in the optic nerve head by laser speckle flowgraphy and scanning laser Doppler flowmetry," Am. J. Ophthalmol. 129(6), 734-739 (2000).

18. K. Yaoeda et al., "Measurement of microcirculation in optic nerve head by laser speckle flowgraphy in normal volunteers," Am. J. Ophthalmol. 130(5), 606-610 (2000).

19. Y. Wang et al., "Measurement of total blood flow in the normal human retina using Doppler Fourier-domain optical coherence tomography," Br. J. Ophthalmol. 93(5), 634-637 (2009).

20. B. C. Chauhan and F. M. Smith, "Confocal scanning laser Doppler flowmetry: experiments in a model flow system," J. Glaucoma 6(4), 237245 (1997).

21. T. Kida, J. H. Liu, and R. N. Weinreb, "Effect of aging on nocturnal blood flow in the optic nerve head and macula in healthy human eyes," J. Glaucoma 17(5), 366-371 (2008).

22. B. Pemp et al., "Diurnal fluctuation of ocular blood flow parameters in patients with primary open-angle glaucoma and healthy subjects," $B r . J$. Ophthalmol. 93(4), 486-491 (2009).

23. A. Luksch et al., "Twelve-hour reproducibility of retinal and optic nerve blood flow parameters in healthy individuals," Acta Ophthalmol. 87(8), 875-880 (2009).

24. R. K. Wang, "In vivo volumetric blood flow imaging using optical microangiography at capillary level resolution," in Conf. Proc. IEEE Engineering Medicine Biology Society 2008, p. 804 (2008).

25. R. K. Wang and L. An, "Doppler optical micro-angiography for volumetric imaging of vascular perfusion in vivo," Opt. Express 17(11), 8926-8940 (2009).

26. R. K. Wang, "Optical microangiography: a label free 3D imaging technology to visualize and quantify blood circulations within tissue beds in vivo," IEEE J. Sel. Top Quantum Electron 16(3), 545-554 (2010).

27. R. K. Wang et al., "Optical microangiography provides depth-resolved images of directional ocular blood perfusion in posterior eye segment," J. Biomed. Opt. 15(2), 020502 (2010).

28. R. K. Wang et al., "Depth-resolved imaging of capillary networks in retina and choroid using ultrahigh sensitive optical microangiography," Opt. Lett. 35(9), 1467-1469 (2010).

29. L. An, T. T. Shen, and R. K. Wang, "Using ultrahigh sensitive optical microangiography to achieve comprehensive depth resolved microvasculature mapping for human retina," J. Biomed. Opt. 16(10), 106013 (2011).

30. M. R. Thorell et al., "Swept-source OCT angiography of macular telangiectasia type 2," Ophthalmic Surg. Lasers Imaging Retina 45(5), 369380 (2014).
31. S. Yousefi, J. Qin, and R. K. Wang, "Super-resolution spectral estimation of optical micro-angiography for quantifying blood flow within microcirculatory tissue beds in vivo," Biomed. Opt. Express 4(7), 1214-1228 (2013).

32. S. Yousefi and R. K. Wang, "Simultaneous estimation of bidirectional particle flow and relative flux using MUSIC-OCT: phantom studies," Phys. Med. Biol. 59(22), 6693 (2014).

33. Q. Zhang et al., "Wide-field imaging of retinal vasculature using optical coherence tomography-based microangiography provided by motion tracking," J. Biomed. Opt. 20(6), 066008 (2015).

34. X. Yin, J. R. Chao, and R. K. Wang, "User-guided segmentation for volumetric retinal optical coherence tomography images," J. Biomed. Opt. 19(8), 086020 (2014).

35. R. Reif et al., "Quantifying optical microangiography images obtained from a spectral domain optical coherence tomography system," Int. J. Biomed. Imaging 2012, 9 (2012).

36. A. F. Frangi et al., Multiscale Vessel Enhancement Filtering, pp. 130137, Springer, New York City (1998).

37. P. E. Shrout and J. L. Fleiss, "Intraclass correlations: uses in assessing rater reliability," Psychol. Bull. 86, 420-428 (1979).

38. J. L. Fleiss, The Design and Analysis of Clinical Experiments, pp. 1-32, John Wiley \& Sons, New York (1986).

39. Y. Jia et al., "Optical coherence tomography angiography of optic disc perfusion in glaucoma," Ophthalmology 121(7), 1322-1332 (2014).

40. X. Wang et al., "Correlation between optic disc perfusion and glaucomatous severity in patients with open-angle glaucoma: an optical coherence tomography angiography study," Graefes Arch. Clin. Exp. Ophthalmol. 253(9), 1557-1564 (2015).

41. Y. Yasuno et al., "In vivo high-contrast imaging of deep posterior eye by 1-microm swept source optical coherence tomography and scattering optical coherence angiography," Opt. Express 15(10), 6121-6139 (2007).

42. K. Takayama et al., "Three-dimensional imaging of lamina cribrosa defects in glaucoma using swept-source optical coherence tomography," Invest. Ophthalmol. Vis. Sci. 54(7), 4798-4807 (2013).

Chieh-Li Chen received her BS degree in electrical engineering and her MS degree in electronics engineering from the National Taiwan University in 2008 and 2010, respectively, and her PhD in bioengineering from the University of Pittsburgh in 2014. She is a senior research fellow at the University of Washington, Department of Bioengineering. Her research interests include medical image processing and data analysis, especially for optical coherence tomography (OCT) and OCT-based angiography images.

Biographies for the other authors are not available. 